\title{
1 Virion-associated spermidine transmits with Rift Valley fever virus 2 particles to maintain infectivity
}

4 Vincent Mastrodomenico ${ }^{1}$, Jeremy J. Esin ${ }^{1,2}$, Shefah Qazi ${ }^{3}$, Oreoluwa S. Omoba ${ }^{1,2}$, Brittany L.

5 Fung $^{1}$, Maxim A. Khomutov ${ }^{4}$, Alexander V. Ivanov ${ }^{4}$, Suchetana Mukhopadhyay ${ }^{3}$, Bryan C.

6 Mounce $^{1,2 *}$

${ }^{1}$ Department of Microbiology and Immunology, Stritch School of Medicine, Loyola University 9 Chicago, Maywood, IL 60153 USA.

$10{ }^{2}$ Infectious Disease and Immunology Research Institute, Stritch School of Medicine, Loyola 11 University Chicago, Maywood, Illinois, USA

$12{ }^{3}$ Department of Biology, Indiana University, Bloomington, Indiana, USA

$13{ }^{4}$ Engelhardt Institute of Molecular Biology, Russian Academy of Sciences, Moscow, Russia

14 * To whom correspondence should be addressed:

Department of Microbiology and Immunology Loyola University Chicago, Stritch School of Medicine 2160 S. First Ave.

Maywood, IL 60153

Short Title: Rift Valley fever virions associate with spermidine

22 Keywords: bunyaviruses, spermidine, polyamines, virus infectivity

7082163358 , bmounce@luc.edu

\section{Abstract}

Viruses require host cell metabolites to productively infect, and the mechanisms by which viruses usurp these molecules is diverse. One group of cellular metabolites important in virus infection is the polyamines, small positively-charged molecules involved in cell cycle, translation, and nucleic acid synthesis, among other cellular functions. Polyamines also support replication of diverse viruses, and they are important for processes such as transcription, translation, and viral protein enzymatic activity. Rift Valley fever virus (RVFV) is a negative-sense RNA virus that requires polyamines to produce infectious particles. In polyamine depleted conditions, noninfectious particles are produced that interfere with virus replication and stimulate immune signaling. Here, we find that RVFV relies on virion-associated polyamines to maintain infectivity. 
Mastrodomenico et al.

34 We show that RVFV replication is facilitated by any of the three biogenic polyamines; however,

35 we specifically find spermidine associated with purified virions. Using a panel of polyamine

36 homologs, we observe that virions can also associate with $(R)$-3-methylspermidine and

37 norspermidine, though not with other less homologous molecules. Using polyamine reporter

38 cells, we demonstrate that virion-associated polyamines transmit from one infected cell to

39 another. Finally, we find that virions devoid of polyamines are unstable and cannot be

40 supplemented with exogenous polyamines to regain stability or infectivity. These data highlight

41 a unique role for polyamines, and spermidine in particular, in maintaining virus infectivity, a

42 function not previously appreciated. Further, these studies are the first to identify polyamines

43 associated with RVFV virions. Targeting polyamines represents a promising antiviral strategy,

44 and this work highlights a new mechanism by which we can inhibit virus replication through

45 FDA-approved polyamine depleting pharmaceuticals.

\section{Introduction}

48 Rift Valley fever virus (RVFV) is a significant human and ruminant pathogen, associated with

49 hemorrhagic fever and spontaneous abortion. While the virus is currently geographically

50 restricted to Africa and the Middle East, the potential for spread globally is significant. Further,

51 RVFV is a mosquito-borne virus, and chikungunya ${ }^{1}$ and Zika $^{2}$ virus demonstrate that these

52 viruses can spread globally and explosively. Both Culex and Aedes species of mosquitoes

53 transmit $\mathrm{RVFV}^{3-5}$, though the breadth of vectors susceptible to RVFV is not fully understood.

54 Fortunately, several vaccine candidates ${ }^{6-8}$ show promise in reducing transmission, including in

55 animals. However, adverse events associated with these vaccines have limited their use, and

56 the virus continues to present itself in frequent outbreaks ${ }^{9-11}$, infecting hundreds and severely

57 impacting local economies. Thus, the development of improved vaccines or the identification of

58 novel antiviral targets is essential to the treatment and prevention of RVFV.

As obligate intracellular pathogens, viruses rely on their host cells for the building blocks of replication. These building blocks include a variety of metabolites produced by the host cell. One set of these metabolites crucial to virus replication is the family of polyamines. Eukaryotic

63 cells synthesize polyamines to support transcription, translation, and cell cycling ${ }^{12-14}$. The 64 biogenic polyamines include putrescine, spermidine, and spermine, which are maintained at 65 millimolar level within cells ${ }^{15}$ and readily interconvert within cells ${ }^{16}$. These molecules are carbon 66 chains of increasing length with primary and secondary amine groups. At physiological $\mathrm{pH}$, 67 polyamines are positively charged, which facilitates nucleic acid interactions. In fact, upwards of 
Mastrodomenico et al.

$6885 \%$ of polyamines are bound to nucleic acids (primarily RNA), proteins, or lipids to support

69 cellular functions ${ }^{17}$. However, polyamines are dispensable for cellular homeostasis in non-

70 transformed cells. Depletion of polyamines via the specific inhibitor difluoromethylornithine

71 (DFMO) reduces cellular proliferation but is otherwise nontoxic ${ }^{18}$. In humans, chronic DFMO

72 treatment has mild side effects and is used in the treatment of trypanosomiasis ${ }^{19,20}$. Additionally,

73 diethylnorspermidine (DENSpm) is a nontoxic molecule that enhances polyamine catabolism by

74 acetylating polyamines and promoting their export or degradation. Thus, while polyamines are

75 crucial to cellular replication, organismal polyamine depletion is tolerable.

77 Early work demonstrated that a subset of viruses incorporate polyamines in virions, especially

78 large DNA viruses like herpesviruses and vaccinia virus, which use polyamines to package their 79 large dsDNA genomes ${ }^{21-23}$. In contrast, RNA viruses, with relatively smaller single-stranded 80 genomes were poorly studied in the context of polyamine metabolism. Polyamines facilitate 81 RNA virus replication, and the polyamine inhibitor DFMO reduces the replication of diverse RNA 82 viruses, including alphaviruses, flaviviruses, enteroviruses, and bunyaviruses, both in vitro and 83 in vivo ${ }^{24,25}$. Chikungunya and Zika viruses (CHIKV and ZIKV) rely on polyamines for genome 84 replication and viral polyprotein translation ${ }^{24}$. Additionally, we have shown that polyamines 85 enhance viral protease activity ${ }^{26}$, promote infectious particle production ${ }^{27}$, and support virus-cell 86 binding ${ }^{28}$ in enteroviruses and bunyaviruses. The breadth of mechanisms by which polyamines 87 support virus infection remain unknown but recent evidence suggests that different viruses utilize polyamines via different mechanisms.

The distinct structures of polyamines have different roles in cells and in viruses. For instance, spermidine copurifies with E.coli tRNAs ${ }^{29}$ and each biogenic polyamine has a distinct affinity for

92 tRNA $^{30}$. Spermidine is also used specifically in the genesis of the modified amino acid hypusine, 93 which is important for translation ${ }^{31,32}$ and also crucial in the replication of some viruse ${ }^{33,34}$. 94 Herpesviruses preferentially package spermidine and spermine but not putrescine in their 95 virions ${ }^{21}$, though it is unknown why these polyamines are preferred. In contrast, chikungunya 96 virus polymerase is stimulated by polyamines and was equally stimulated by putrescine, 97 spermidine, or spermine ${ }^{24}$, suggesting that some viral processes may be insensitive to 98 polyamine identity. Similarly, phage T7 polymerase is stimulated by spermidine and a variety of 99 polyamines not synthesized in eukaryotic or prokaryotic cells ${ }^{13}$. 
Mastrodomenico et al.

101 Polyamines are crucial to RVFV infection, and we demonstrated that polyamine depletion

102 reduces RVFV titers and leads to the production of noninfectious particles that interfere with 103 virus replication ${ }^{27}$. Precisely how polyamines function in RVFV infection remains unclear, 104 however. Here, we investigated whether RVFV relied on specific polyamines for replication. We 105 observe that RVFV replication is supported by any of the biogenic polyamines as well as 106 cadaverine and norspermidine, two bacterially-synthesized polyamines. We find that these 107 polyamines support infectious particle production. We considered that polyamines may be 108 associated with RVFV virions and measured them via fluorometric assay and thin layer 109 chromatography. We identify spermidine within purified virions and that single-carbon 110 modifications of spermidine can also associate with virions. We finally show that virion111 associated spermidine enhances viral particle infectivity and that exogenous polyamines applied 112 to virions cannot restore their infectivity. In sum, polyamines, spermidine in particular, are crucial 113 to RVFV due to their association with the virion which maintains infectivity.

115 Results

116 Rift Valley fever virus is sensitive to low concentrations of biogenic polyamines. 117 Bunyaviruses are sensitive to polyamine depletion mediated either by DFMO or DENSpm, and 118 replenishing polyamines exogenously fully rescues replication. To determine if specific 119 polyamines enhance virus replication, we depleted Huh7 cells of polyamines using $1 \mathrm{mM}$ 120 DFMO, infected at multiplicity of infection (MOI) of 0.1 plaque-forming units (pfu) per cell with 121 RVFV strain MP-12 and then titrated the biogenic polyamines putrescine, spermidine, and 122 spermine at the time of infection. After $48 \mathrm{~h}$, virus was collected and titered by plaque assay on 123 Vero-E6 cells. We observed that DFMO significantly reduced viral titers compared to untreated 124 samples, not supplemented with DFMO or exogenous polyamines (Figure 1, dashed line 125 "DFMO" versus not treated, or "NT"). We further observed that viral titers remained suppressed 126 until polyamine concentration passed $1 \mu \mathrm{M}$, which held for each of putrescine, spermidine and 127 spermine (Figure 1A). We observed $\mathrm{EC}_{50}$ values of 3.6, 4.6, and $9.9 \mu \mathrm{M}$ for spermine, 128 spermidine, and putrescine, respectively. In fact, each polyamine rescued viral titers to levels 129 that were not significantly different from untreated samples at $10 \mu \mathrm{M}$. We performed a similar 130 analysis with distantly-related bunyavirus La Crosse virus (LACV) and observed similar results: 131 all three biogenic polyamines supported infection in the micro-molar range (Figure 1B) and no 132 polyamine was favored when supplemented to DFMO-treated cells. 
Mastrodomenico et al.

Polyamines not synthesized in eukaryotes also support viral replication. The biogenic polyamines are found in all eukaryotic cells examined. Given the relatively simple structure of polyamines, consisting of carbon chains with primary and secondary amines, we hypothesized that additional non-biogenic molecules with similar structures may also enhance virus replication. To test this, we treated Huh7 cells with $1 \mathrm{mM}$ DFMO, infected with RVFV, and supplemented cells with an array of polyamines at $10 \mu \mathrm{M}$ at the time of infection (a selection of structures shown in Figure 1C. All polyamines except putrescine, spermidine, and spermine are not synthesized in eukaryotic cells and are non-biogenic). When we measured viral titers at 48 hpi, we observed that biogenic polyamines fully rescued viral titers; however, we also observed that the polyamines cadaverine (1,4-diaminopentane, one carbon longer than putrescine) and norspermidine (one fewer carbon than spermidine), two polyamines not synthesized by eukaryotic cells, rescued viral titers to nearly equivalent levels as the biogenic polyamines (Figure 1D). Interestingly, we observed that no other non-biogenic polyamines enhanced titers beyond DFMO treatment levels, despite their structural similarity. Of particular interest, butylamine, which is similar to putrescine but lacks an amino group, failed to enhance replication. Additionally, elongating the putrescine carbon chain to six (diaminohexane) or seven (diaminoheptane) carbons or shortening the chain to three carbons also eliminated enhancement of virus replication. Again, we tested this set of polyamines with LACV and observed that cadaverine and norspermidine again enhanced viral titers, while all other compounds did not, suggesting conservation in polyamine usage between these two bunyaviruses (Figure 1E).

Finally, we used an enterovirus model of infection, Coxsackievirus B3 (CVB3) to test its sensitivity to distinct polyamines. As with RVFV and LACV, we treated cells with DFMO and supplemented with polyamines at the time of infection. When we titrated the three biogenic polyamines, we observed enhancement of replication at $1 \mu \mathrm{M}$ of either putrescine, spermidine, or spermine (Figure 1F), similar to RVFV and LACV. We also tested whether CVB3 could utilize cadaverine or norspermidine for replication. When we supplemented DFMO-treated cells with either of these compounds, however, neither cadaverine nor norspermidine enhanced replication (Figure 1G). These data suggest that distinct virus families may rely on distinct 164 polyamine structures for optimal replication. 
Mastrodomenico et al.

168

169

170

171

172

173

174

175

176

177

178

179

180

181

182

183

184

185

biogenic polyamines also maintain specific infectivity of RVFV, we measured the ratio of genomes to infectious virus, as measured by plaque assay. We treated Huh7 cells with $1 \mathrm{mM}$ DFMO prior to infection at $\mathrm{MOI} 0.1$. At the time of infection, we added $100 \mu \mathrm{M}$ putrescine, spermidine, or spermine. After $48 \mathrm{~h}$, supernatant was collected for titration and RNA purification. RNA was reverse transcribed and analyzed for RVFV genomes using virus-specific primers. We then calculated the genome-to-PFU ratio as a measure of specific infectivity. Similar to our previous work, polyamine depletion increased the ratio of genomes to PFU (Figure 2A), suggesting reduced specific infectivity. However, addition of putrescine or spermine returned this ratio to untreated levels and modestly increased specific infectivity (fewer genomes per PFU) with spermidine treatment. We performed a similar analysis for LACV and observed the same phenotype: DFMO treatment increases the genome-to-PFU ratios, while the biogenic polyamines reduce to untreated levels (Figure $2 \mathrm{~B}$ ).

Measuring genome-to-PFU is a surrogate for measuring the number of viral particles compared to the number of these particles that are infectious. To more accurately measure particle-to-PFU ratio, we used a method similar to Wichgers Schreur and colleagues to stain viral particles fluorescently ${ }^{35}$. We spinoculated virus on coverslips and stained for viral envelope glycoprotein Gc or Gn using specific antibodies and FITC-tagged fluorescent secondary antibody. To establish the assay, we tested both antibodies to ensure specificity and that we weren't observing aberrations due to impurities on the coverslips or nonspecific staining. Using spinoculated virus derived from infected and uninfected cells, we observed dots corresponding to virus in only samples that were infected, no detectable signal was observed on slides spinoculated with samples from uninfected cells (Figure 2C). We next applied this method to virus derived from DFMO-treated cells as well as cells supplemented with various polyamines. Again, virus was spinoculated from mock- or virus-infected cell supernatant and stained with anti-Gn and FITC-tagged secondary. As a control, we also stained with a secondary antibody fluorescent in the red channel (TxRed). When we visualized the samples, we observed distinct puncta only in infected samples and not in mock samples (Figure 2D). We also observed no staining in the red channel, suggesting that we were again not observing impurities on the coverslips (Figure 2D, "Red channel"). We counted the number of dots using ImageJ and backcalculated the number of particles per $\mathrm{mL}$ of infected cell supernatant (Figure $2 \mathrm{E}$ ). We used this number to calculate the particle-to-PFU ratio (Figure 2F). Supporting our genome-to-PFU ratios, we observed that untreated cells had a paticle-to-PFU ratio of approximately 20 , and DFMO-mediated polyamine depletion increased this to $>300$. Thus, DFMO treatment increases 
Mastrodomenico et al.

202 the genome-to-PFU ratio, as well as the particle-to-PFU ratio, as measured by our two methods. 203 To expand our rescue experiments, we similarly stained particles derived from infection of 204 DFMO-treated and polyamine-supplemented cells. As with our genome-to-PFU ratio, we 205 observe that addition of any of the biogenic polyamines returns the particle-to-PFU ratio to 206 untreated levels, with a small, though significant, reduction in this ratio, suggesting that 207 polyamines support RVFV infectivity.

Polyamines are associated with the RVFV virion. We observe a change in specific infectivity (as measured by genome-to-PFU or particle-to-PFU), and we previously characterized that the physical and structural properties of virions produced with or without polyamines are indistinguishable. We next considered that polyamines might be associated with RVFV virions themselves. In fact, polyamines are found in the virions of several DNA viruses and a subset of RNA viruses. To this end, we used a fluorometric assay, which directly measures polyamine content in cells. We generated virus in Huh7 cells left untreated or treated with $1 \mathrm{mM} \mathrm{DFMO}$ by infecting at $\mathrm{MOI} 0.1$ for $48 \mathrm{~h}$. After $48 \mathrm{~h}$, we purified virus by sucrose cushion ultracentrifugation, resuspended the viral pellet in PBS, and analyzed polyamine association. As a control, we used mock-infected supernatant and performed all steps in tandem with virus-infected cell supernatant. As expected, our purified mock-infected supernatant had no signal (Figure 3A). Similarly, CVB3-infected cell supernatant exhibited no detectable signal above background, as expected $^{23}$. As a positive control ${ }^{22}$, we observed detectable levels of polyamines in purified vaccinia virus (VACV), and this signal returned to background levels when virus was derived from DFMO-treated conditions. When we tested RVFV, we observed signal above background, though not as intense as VACV, and this signal was depleted when virus was derived from DFMO-treated cells. These results suggest that purified RVFV virions are associated with polyamines. cell; thus, this assay could not dictate which specific polyamine is associated with virions. In order to identify the polyamine(s), we purified and concentrated RVFV from Huh7 cells (approximately $10^{6} \mathrm{PFU}$ total) as above, labeled polyamines via dansylation, and resolved the 232 dansylated polyamines via thin layer chromatography (TLC). When we analyzed mock-infected 233 cell supernatant, we observed no polyamines, as expected (Figure 3B). The whole cell lysate 234 (WCL) from cells infected with RVFV contained robust amounts of spermidine and spermine, 235 though little putrescine was detected. Interestingly, purified RVFV virions exhibited a distinct 
Mastrodomenico et al.

band corresponding to spermidine, and this band was lost when virus was purified from DFMOtreated cells. LACV also showed virion-associated spermidine, while human rhinovirus serotype 2 (HRV2, enterovirus distantly related to CVB3) had no detectable polyamines, as expected. These data again detect virion-associated polyamines, which we have identified as spermidine.

Polyamines interconvert upon replenishment of DFMO-treated cells. We next considered whether we could deplete polyamines from cells, replenish with individual biogenic polyamines

243 and detect these species in the RVFV virion. To this end, we treated Huh7 cells with $1 \mathrm{mM}$

244 DFMO, infected with RVFV at MOI 0.1, and added $10 \mu \mathrm{M}$ putrescine, spermidine, or spermine 245 individually at the time of infection. As a control, we added ornithine, the polyamine precursor. 246 As before, we purified and concentrated virions and analyzed polyamine content by thin layer 247 chromatography. As expected, virions purified from DFMO-treated cells exhibited no 248 polyamines; however, polyamine supplementation resulted in detectable virion-associated 249 polyamines (Figure 4A). Interestingly, both putrescine and spermine supplementation led to a detectable level of virion-associated spermidine. Given that DFMO blocks the production but not the interconversion of polyamines, we considered that supplemented putrescine and spermine might generate spermidine through the actions of spermidine synthase (SMS) or polyamine oxidase (PAOX) with spermidine/spermine acetyltransferase (SAT1). We measured polyamine levels in DFMO-treated cells that were supplemented with the polyamines and observed that with putrescine, spermidine, or spermine supplementation, spermidine was abundant on our TLC (Figure 4B). Thus, the biogenic polyamines rapidly interconvert and specifically spermidine is virion associated in this polyamine milieu.

While eukaryotic cells can interconvert the biogenic polyamines, no description of their ability to interconvert cadaverine or norspermidine has been reported. Thus, we tested whether supplementation of these polyamines, which rescues viral titers, can support polyamine packaging. To this end, we generated and purified virus from DFMO-treated cells supplemented with $10 \mu \mathrm{M}$ cadaverine or norspermidine and measure virion-associated polyamines by TLC. Curiously, we detected bands near the retention factor (Rf) of spermidine, though not precisely at spermidine's Rf (Figure 4C). Our standards (Figure 4C, right) suggest that norspermidine is, in fact, associated with RVFV virions. However, the band in the cadaverine lane was faint and migrated slightly higher in the chromatogram. This band could be $\mathrm{N}$ (3-aminopropyl)cadaverine, which is a single carbon longer than spermidine, though this molecule has not been described

269 to be synthesized in human cells. We checked whether we could detect this species in cells 
Mastrodomenico et al.

270 (Figure 4D), and we can in fact a molecule that runs as N(3-aminopropyl)cadaverine would.

271 However, we also observe that norspermidine is not interconverted into other detectable

272 polyamine species. In sum, however, it appears that polyamines within a limit can replace

273 spermidine for RVFV virions.

Methylated spermidine supports RVFV replication and is virion-associated. Polyamines rapidly interconvert, as observed (Figure 2B) and previously described. Methylated spermidine and spermine are not good substrates for acetylation by spermidine/spermine acetyltransferase and their interconversion is limited. (R)-3-methylspermidine is afunctionally active and metabolically stable analog of biogenic spermidine. However, $(R)$-3-methylspermidine is a poor substrate for spermine synthase and SAT $1^{36}$. Thus, we considered whether methylated spermidine could enhance viral replication in the absence of biogenic spermidine and if this modified polyamine could be virion-associated. To test this, we treated cells with DFMO and replenished with $(R)$-3-methylspermidine (Figure $5 \mathrm{~A}$ ) at the time of infection with RVFV. When we measured titers at $48 \mathrm{hpi}$, we observed a full rescue in titers, to a level similar to spermidine. We titrated $(R)$-3-methylspermidine after DFMO treatment and observed that concentrations around $10 \mu \mathrm{M}$ were sufficient to fully rescue viral titers (Figure $5 \mathrm{~B}$ ), slightly higher than for spermidine (Figure 1A). Thus, methylated spermidine functions to support RVFV infection.

To test if $(R)$-3-methylspermidine could associate with virions, we purified virions and visualized polyamines by TLC. As expected, we observed that RVFV was associated with spermidine; however, we could detect bands corresponding to $(R)$-3-methylspermidine as well (Figure 5D), suggesting that this polyamine is virion-associated. To confirm that 3-methylspermidine was not interconverted to the biogenic polyamines, we also performed TLC on the treated cells and observed no such interconversion (Figure 5E).

We previously showed that virions derived from DFMO-treated cells show no distinctions in their gross appearance by electron microscopy. To confirm this phenotype as well as to determine whether polyamine rescue with 3-methylspermidine could change virion morphology, we purified virions and examined them by electron microscopy. In untreated conditions, we observed 300 numerous virions of expected size and with visible surface glycoproteins (Figure 5F). As 301 previously described, DFMO treatment did not noticeably change virion appearance, and 302 spermidine or 3-methylspermidine supplementation to DFMO-treated cells similarly had no 
Mastrodomenico et al.

discernible effect on virion appearance. Thus, polyamines do not appear to contribute to virion morphology.

Polyamines are transmitted to naïve cells upon infection. Given that polyamines, specifically spermidine, are associated with RVFV virions, we considered that virus infection may transmit polyamines to newly-infected cells. We generated virus stock from untreated and DFMO-treated cells (Figure 6A) and then used these viruses to infect polyamine-sensitive luciferase reporter cells. These reporter cells consist of 293T cells transfected with an OAZ1 dual-luciferase construct. Briefly, OAZ1 transcript is sensitive to cellular polyamine levels: high polyamines result in enhanced stability and translation; low polyamines result in reduced stability and translation. With this construct, we can measure luciferase activity to indirectly measure polyamine levels in cells: polyamine levels directly correlate with firefly luciferase activity, which is normalized to renilla luciferase activity that is polyamine-independent. We treated these reporter cells with DFMO and observed a significant reduction in luciferase activity, corresponding to reduced polyamine levels. To these DFMO-treated reporter cells, we added $10 \mu \mathrm{M}$ polyamines (equimolar mixture of the biogenic polyamines) and observed enhanced luciferase activity, demonstrating their responsiveness to polyamines. We also added purified RVFV virions $\left(10^{3}, 5 \times 10^{3}\right.$, and $\left.10^{4} \mathrm{PFU}\right)$, for which we observed a dose-dependent increase in signal (Figure 6B). When we added virus $\left(10^{4} \mathrm{pfu}\right)$ derived from DFMO-treated cells or when we purified supernatant from mock-infected cells, we did not observe an increase in luciferase activity, indicating that we are not observing a cellular response to infection or aberrantly purifying polyamines from cellular supernatant. In sum, these data suggest that RVFV can transmit polyamines upon infection. and polyamine depletion results in the genesis of non-infectious particles. Additionally, we can detect spermidine (and highly similar non-biogenic polyamines) in purified RVFV. We hypothesized that these non-infectious particles may be due to a decline in viral infectivity from a lack of virion-associated polyamines. To test this, we generated virus from untreated and DFMO-treated Huh7 cells and incubated the cell-free supernatant at $37^{\circ} \mathrm{C}$ for $24 \mathrm{~h}$, taking

333 samples at regular intervals to titer. We observed that virus derived from untreated cells slowly 334 declined in titer (Figure 7A), resulting in a modest reduction in titers over $24 \mathrm{~h}$. The calculated 335 half-life of the virus was approximately $29.4 \mathrm{~h}$. In contrast, virus derived from DFMO-treated cells 
Mastrodomenico et al.

rapidly lost infectivity, with titers dropping by about $90 \%$ within $24 \mathrm{~h}$ and a half-life of approximately $14.4 \mathrm{~h}$. Thus, polyamine-depleted cells generate virus that rapidly loses infectivity.

We first considered that DFMO itself was destabilizing RVFV virions. Thus, we incubated virus with $1 \mathrm{mM}$ or $500 \mu \mathrm{M}$ DFMO for $24 \mathrm{~h}$ at $37^{\circ} \mathrm{C}$. We observed that DFMO itself did not destabilize the virus, as titers were equivalent between untreated and DFMO-treated viruses (Figure 7B)

342 Because spermidine is found in association with purified RVFV virions, we next hypothesized 343 that polyamines themselves may stabilize virions. To this end, we incubated virus generated 344 from untreated or DFMO-treated cells with $10 \mu \mathrm{M}$ spermidine or spermine and incubated at $34537^{\circ} \mathrm{C}$ for $24 \mathrm{~h}$. We observed decay of the virus derived from DFMO-treated cells accelerated 346 compared to untreated cells, with no difference regardless of polyamine treatment (Figure 7C).

347 These data suggest that polyamines do not stabilize RVFV when added exogenously. Together, 348 these data suggest that polyamines help to maintain infectivity, that this is not due to DFMO itself, and that exogenous polyamines cannot stabilize virions.

To extend these results to the related bunyavirus LACV, we similarly incubated virus derived from untreated and DFMO-treated conditions and measured infectivity over 24h. As with RVFV, we observed a steep decline in virus titers, though the effect was primarily at 48 hpi (Figure 7D).

354 Thus, LACV exhibits similar sensitivity to losing infectivity when the virus is derived from DFMOtreated cells.

Finally, we considered whether we could potentially resurrect infectivity of our viral particles by incubating them with polyamines, specifically spermidine. To this end, we incubated virus from untreated and DFMO conditions with increasing doses of spermidine for $24 \mathrm{~h}$ at $37^{\circ} \mathrm{C}$. We observed no significant difference in titer from spermidine treatment (Figure 7E), suggesting that spermidine supplementation cannot rescue infectivity of the virions once they have lost infectivity.

\section{Discussion}

365 As obligate intracellular pathogens, viruses rely on the host to provide metabolites for replication. The virion itself is composed of molecules derived from the host but directed for assembly and order by the viral genome. The genomic nucleotides, proteins' amino acids, and envelope's lipids originate from host metabolites. Here, we identify polyamines as an additional 
Mastrodomenico et al.

370 polyamines in the virions of herpesviruses ${ }^{21}$ and poxviruses ${ }^{22}$. However, other viruses do not 371 appear to consistently incorporate polyamines into virions. For example, negligible amounts of 372 polyamines are detected associated with poliovirus capsids, but rhinovirus 14 had a detectable 373 amount, enough to neutralize approximately $16 \%$ of the genome ${ }^{23}$. Adenovirus-5, a DNA virus, 374 also appears to incorporate small amounts of polyamines ${ }^{37}$. Thus, the packaging of polyamines 375 and the role(s) of these packaged polyamines is not necessarily evolutionarily conserved. 376 Additionally, the presence of these polyamines had not been examined in bunyaviruses.

We specifically observe that spermidine is associated with RVFV and LACV virions, despite cellular polyamine pools comprising primarily spermidine and spermine. Other viruses incorporate polyamines without specificity; for example, densoviruses have all three polyamines in purified virus ${ }^{38}$. Interestingly, herpesviruses package spermidine and spermine, with spermidine primarily associated with the envelope or tegument and spermine in the viral capsid $^{21}$. Given that bunyaviruses like RVFV do not have a bona fide capsid, one could speculate that the viral envelope specifically associates with spermidine, given that both viruses have a lipid membrane component. The localization of polyamines in RVFV may further inform the function of these polyamines in the virion. Additionally, the mechanism by which virions incorporate spermidine but exclude the other polyamines is not known for this or other viruses. Whether spermidine incorporation is an active process by the virus or a product of RNA-, protein-, or lipid-spermidine interactions will be an important distinction to make.

Despite the specific association of spermidine with purified virions, we observe that any of the biogenic polyamines (putrescine, spermidine, and spermine) supports viral infection. Importantly, when treating cells with these polyamines, they interconvert and produce the full complement of cellular polyamines. In fact, we observe this in our system, and those cells replenished with any of the biogenic polyamines support virus infection and spermidine association with virions. Thus, the balance of polyamines is crucial to cellular homeostasis ${ }^{16}$ and virus replication. Interestingly, RVFV exhibits some flexibility in polyamine utilization, as we can detect molecules that are a single carbon longer or shorter than spermidine in virions. Interestingly, cells exhibit heterogeneity in their polyamine composition and, thus, if viruses

400 differentially utilize polyamines, the cellular polyamine composition may alter infection and 401 pathogenesis. Regardless, whether distinct polyamines function differently during RVFV 402 infection remains to be fully understood. 
Mastrodomenico et al.

404 We previously observed that polyamine depletion led to the accumulation of noninfectious viral 405 particles that interfered with productive virus infection ${ }^{27}$. We were unable to find a physical 406 distinction between infectious and noninfectious virions, however. These studies suggest that a 407 component of the virus that may be maintaining infectivity is spermidine, as viruses lacking 408 polyamines are more labile than viruses propagated in cells with polyamines. However, these 409 results do not preclude that an additional polyamine-modulated factor may contribute to virion 410 stability. In fact, polyamine depletion affects several cellular processes. Regardless, we observe 411 that polyamine depletion generates viral particles lacking polyamines that rapidly lose infectivity.

412 Future work will address the mechanisms behind this lability.

Diverse viruses rely on polyamines for their replication, and the diversity of viruses may rely on different polyamines for different processes. For example, Ebolavirus, a filovirus, utilizes polyamines for genome replication but hypusine, derived from spermidine, for protein translation $^{33,34}$. As mentioned, herpesviruses package spermidine in viral envelope/tegument and spermine in capsids ${ }^{21}$. Whether these phenotypes are broadly shared is unclear, but understanding the mechanisms by which viruses utilize polyamines may highlight both evolutionarily conserved and divergent mechanisms. Importantly, however, the requirement of polyamines for productive infection is broadly shared ${ }^{25}$, and targeting polyamine metabolism through host-directed antivirals represents a promising means of blocking virus infection.

\section{Materials and Methods}

425 Cell culture. Cells were maintained at $37 \square \mathrm{C}$ in $5 \% \mathrm{CO}_{2}$, in Dulbecco's modified Eagle's medium (DMEM; Life Technologies) with bovine serum and penicillin-streptomycin. Vero cells (BEI Resources) were supplemented with 10\% new-born calf serum (NBCS; Thermo-Fischer) and Huh7 cells, kindly provided by Dr. Susan Uprichard, were supplemented with $10 \%$ fetal bovine serum (FBS; Thermo-Fischer).

Drug treatment. Difluoromethylornithine (DFMO; TargetMol) and N1,N11-Diethylnorspermine (DENSpm; Santa Cruz Biotechnology) were diluted to $100 \mathrm{x}$ solution (100mM and $10 \mathrm{mM}$, respectively) in sterile water. For DFMO treatments, cells were trypsinized (Zymo Research) 434 and reseeded with fresh medium supplemented with $2 \%$ serum. Following overnight 435 attachment, cells were treated with $100 \mu \mathrm{M}, 500 \mu \mathrm{M}, 1 \mathrm{mM}$, or $5 \mathrm{mM}$ DFMO. Cells were 436 incubated with DFMO for 96 hours to allow for depletion of polyamines in Huh7 cells. For 437 DENSpm treatment, cells were treated with $100 \mathrm{nM}, 1 \mu \mathrm{M}, 10 \mu \mathrm{M}, 100 \mu \mathrm{M}$, and $1 \mathrm{mM} 16$ hours 
Mastrodomenico et al.

438 prior to infection. During infection, media was cleared and saved from the cells. The same

439 medium containing DFMO and DENSpm was then used to replenish the cells following

440 infection. Cells were incubated at the appropriate temperature for the duration of the infection.

441 Polyamines (Sigma-Aldrich) were added to cells at the time of infection. Methylated spermidine

442 (3-methylspermidine) was derived as described previously ${ }^{39}$ and were added at the time of

443 infection.

445 Infection and enumeration of viral titers. RVFV MP $-12^{40}$ and LACV were derived from the 446 first passage of virus in Huh7 cells. CVB3 (Nancy strain) was derived from the first passage of 447 virus in Vero-E6 cells. LACV was obtained from Biodefense and Emerging Infections (BEI) 448 Research Resources. For all infections, DFMO and DENSpm were maintained throughout 449 infection as designated. Viral stocks were maintained at $-80 \square$ C. For infection, virus was diluted 450 in serum-free DMEM for a multiplicity of infection (MOI) of 0.1 on Huh7 cells, unless otherwise 451 indicated. Viral inoculum was overlain on cells for 10 to 30 minutes, and the cells were washed 452 with PBS before replenishment of media. Dilutions of cell supernatant were prepared in serum453 free DMEM and used to inoculate confluent monolayer of Vero cells for 30 min at $37 \square$ C. Cells 454 were overlain with $0.8 \%$ agarose in DMEM containing $2 \%$ NBCS. CVB3 samples were 455 incubated for 2 days, RVFV and LACV samples were incubated for 4 days at 37 $\square$. Following 456 appropriate incubation, cells were fixed with $4 \%$ formalin and revealed with crystal violet solution 457 (10\% crystal violet; Sigma-Aldrich). Plaques were enumerated and used to back-calculate the 458 number of plaque forming units (pfu) per milliliter of collected volume.

Virus infectivity assay.

461 RVFV from not-treated or DFMO-treated conditions were incubated at $37^{\circ} \mathrm{C}$ for 24 hours. 462 Subsequent addition of polyamines (10uM spermidine and 10uM spermine) were added to not463 treated or DFMO treated virus and incubated at $37^{\circ} \mathrm{C}$ for 24 hours. LACV virus from not-treated 464 or DFMO treated conditions were incubated at $37^{\circ} \mathrm{C}$ for 52 hours. Supernatant was collected at 465 the indicated time points and viral titer was obtained via plaque assay.

467 Thin layer chromatography determination of polyamines. Polyamines were separated by 468 thin-layer chromatography as previously described ${ }^{41}$. For all samples, cells were treated as 469 described prior to being trypsinized and centrifuged. Pellets were washed with PBS and then 470 resuspended in $200 \mathrm{uL} 2 \%$ perchloric acid. Samples were then incubated overnight at $4 \square$ C. 200 $471 \mathrm{uL}$ of supernatant was combined with $200 \mathrm{uL} 5 \mathrm{mg} / \mathrm{ml}$ dansyl chloride (Sigma Aldrich) in 
Mastrodomenico et al.

472 acetone and $100 \mathrm{uL}$ saturated sodium bicarbonate. Samples were incubated in the dark

473 overnight at room temperature. Excess dansyl chloride was cleared by incubating the reaction

474 with $100 \mu \mathrm{L} 150 \mathrm{mg} / \mathrm{mL}$ proline (Sigma Aldrich). Dansylated polyamines were extracted with 50

$475 \mu \mathrm{L}$ toluene (Sigma Aldrich) and centrifuged. $5 \mu \mathrm{L}$ of sample was added in small spots to the TLC

476 plate (silica gel matrix; Sigma Aldrich) and exposed to ascending chromatography with 1:1

477 cyclohexane: ethylacetate. Plate was dried and visualized via exposure to UV.

Polyamine luciferase reporter assay. To measure free polyamine levels in cells, a dual480 luciferase vector containing the wild-type -1 frameshift antizyme OAZ1 (pC5730) or a dual481 luciferase vector containing an in-frame control (pC6154), kindly sent to us by Dr. Tom Dever 482 from the National Institutes of Health, were transfected into cells. Free polyamines modulate 483 OAZ1 mRNA frameshifting and these constructs can measure relative endogenous polyamine 484 concentrations via a dual-luciferase reporter as previously described ${ }^{42}$. 293T cells were seeded 485 with $2 \%$ media and drug treated as described above. Cells were transfected with $62.5 \mathrm{ng}$ of either pC5730 or pC6154. After 4h, cells were infected where indicated. After 24 hours of incubation, luminescent signal was measured using the Dual-Luciferase Reporter Assay System 488 (Promega) by measuring both firefly and Renilla luciferase with the Veritas Microplate Luminometer (Turner Biosystems). Firefly luciferase was normalized to Renilla and the wild-type values were compared to an in-frame control. These values were normalized to untreated cells as relative light units to obtain relative polyamine content.

RNA purification and cDNA synthesis. Media was cleared from cells and Trizol reagent (Zymo Research) directly added. Lysate was then collected, and RNA was purified according to the manufacturer's protocol utilizing the Direct-zol RNA Miniprep Plus Kit (Zymo Research). Purified RNA was subsequently used for cDNA synthesis using High Capacity cDNA Reverse Transcription Kits (Thermo-Fischer), according to the manufacturer's protocol, with 10-100 ng of RNA and random hexamer primers.

Viral genome quantification. Following cDNA synthesis, qRT-PCR was performed using the 501 QuantStudio3 (Applied Biosystems by Thermo-Fischer) and SYBR green mastermix 502 (DotScientific). Samples were held at 95 $\square$ C for 2 mins prior to 40 cycles of $95 \square \mathrm{C}$ for $1 \mathrm{~s}$ and $50360 \square \mathrm{C}$ for 30s. Primers were verified for linearity using eight-fold serial diluted cDNA and 504 checked for specificity via melt curve analysis following by agarose gel electrophoresis. All 505 samples were used to normalize to total RNA using the $\Delta \mathrm{C}_{\mathrm{T}}$ method. Primers against the RVFV 
Mastrodomenico et al.

small genome were 5'-CAG-CAG-CAA-CTC-GTG-ATA-GA-3' (forward) and 5'-CCC-GGA-GGATGA-TGA-TGA-AA-3'. Primers for LACV small genome were 5'-GGC-AGG-TGG-AGG-TTATCA-AT-3' (forward) and 5'-AAG-GAC-CCA-TCT-GGC-TAA-ATA-C-3' (reverse). GAPDH primers were 5'-GAT-TCC-ACC-CAT-GGC-AAA-TTC-3' (forward) and 5'-CTG-GAA-GAT-GGTGAT-GGG-ATT-3' (reverse).

Genome-to-PFU ratio calculations. The number of viral genomes quantified as described above were divided by the viral titer, as determined by plaque assay, to measure the genometo-PFU ratio. Values obtained were normalized to untreated conditions to obtain the relative genome-to-PFU ratio.

Transmission electron microscopy. Four microliters of purified virus were applied to a 518 Formvar- and carbon-coated 400-mesh copper grid (Electron Microscopy Sciences, Hatfield, 519 PA) for 25 seconds. Sample was removed by blotting, and another $4 \mu \mathrm{L}$ of purified virus was applied for 25 seconds, blotted, and stained with $2 \%$ uranyl acetate for 25 seconds. The stained grids were analyzed using a JEOL 1010 transmission electron microscope (Tokyo, Japan) operating at $80 \mathrm{kV}$. Images were recorded using a Gatan (Pleasanton, CA) UltraScan 4000 charge-coupled-device camera at a magnification of 40,000X.

Spinoculation and indirect immunofluorescence. Virus was spinoculated onto coverslips by centrifugation at $1200 \mathrm{rpm}$ for 2 hours. Coverslips were subsequently fixed with $4 \%$ formalin overnight, washed with PBS, permeabilized and blocked with $0.2 \%$ Triton X-100 and $2 \%$ BSA in PBS (blocking solution) for 60 minutes at room temperature (RT). Cells were sequentially incubated as follows: Primary mouse anti-Gn antibody (1:1000 in blocking solution, overnight at $4^{\circ} \mathrm{C}$ ), and secondary antibody, goat anti-mouse 488nm, (1:1000 in PBS, 2hr, RT). After washing with PBS, cells were mounted with Everbrite Hardset Mounting Medium (Biotium ) overnight. To ensure that signal was not due to impurities, mock-infected supernatant was used as a control and processed in tandem. Samples were imaged with Zeiss Axio Observer 7 with Lumencor

534 Spectra X LED light system and a Hamamatsu Flash 4 camera using appropriate filters using 535 Zen Blue software with a 40X objective (Images were collected with a DeltaVision microscope 536 (Applied Precision) detected with a digital camera (CoolSNAP HQ:Photometrics) with a 60X 537 objective). Images were deconvoluted using SoftWoRx deconvolution software (Applied 538 Precision) and quantified by ImageJ. 
Mastrodomenico et al.

Statistical Analysis. Prism 6 (GraphPad) was used to generate graphs and perform statistical analysis. For all analyses, one-tailed Student's $t$ test was used to compare groups, unless

542 otherwise noted, with $\mathrm{a}=0.05$. For tests of sample proportions, $\mathrm{p}$ values were derived from

543 calculated $Z$ scores with two tails and $\alpha=0.05$.

\section{Acknowledgments}

546 We are gracious to Thomas Gallagher for critical discussion and helpful insights concerning this 547 project. We thank Susan Uprichard for Huh7 cells and Makio Iwashima for THP-1 cells, as well 548 as helpful discussion. We also thank Shinji Makino and Kaori Terasaki for generously providing 549 the MP-12 strain of RVFV. We appreciate immunofluorescent imaging help and input from the 550 labs of Ivana Kuo and Jordan Beach. Synthesis of $(R)$-isomer of 3-MeSpd was supported by 551 grant of Russian Scientific Foundation \#17-74-20049.

\section{References}

554 1. Schuffenecker, I. et al. Genome Microevolution of Chikungunya Viruses Causing the Indian Ocean Outbreak. PLOS Med. 3, e263 (2006).

2. Wikan, N. \& Smith, D. R. Zika virus: history of a newly emerging arbovirus. Lancet Infect. Dis. 16, e119-e126 (2016).

3. Tantely, L. M., Boyer, S. \& Fontenille, D. A Review of Mosquitoes Associated with Rift Valley Fever Virus in Madagascar. Am. J. Trop. Med. Hyg. 92, 722-729 (2015).

4. Sang, R. et al. Distribution and abundance of key vectors of Rift Valley fever and other arboviruses in two ecologically distinct counties in Kenya. PLoS Negl. Trop. Dis. 11, e0005341 (2017).

5. Seufi, A. M. \& Galal, F. H. Role of Culex and Anopheles mosquito species as potential vectors of rift valley fever virus in Sudan outbreak, 2007. BMC Infect. Dis. 10, 65 (2010).

6. Smith, D. R. et al. Attenuation and efficacy of live-attenuated Rift Valley fever virus vaccine candidates in non-human primates. PLoS Negl. Trop. Dis. 12, e0006474 (2018).

7. Ikegami, T. Rift Valley fever vaccines: an overview of the safety and efficacy of the liveattenuated MP-12 vaccine candidate. Expert Rev. Vaccines 16, 601-611 (2017).

8. Faburay, B., LaBeaud, A. D., McVey, D. S., Wilson, W. C. \& Richt, J. A. Current Status of Rift Valley Fever Vaccine Development. Vaccines 5, (2017).

9. Sang, R. et al. Rift Valley Fever Virus Epidemic in Kenya, 2006/2007: The Entomologic Investigations. Am. J. Trop. Med. Hyg. 83, 28-37 (2010). 
Mastrodomenico et al.

573

574

575

576

577

578

579

580

581

582

583

584

585

586

587

588

589

590

591

592

593

594

595

596

597

598

599

600

601

602

603

604

605

606

10. Woods, C. W. et al. An Outbreak of Rift Valley Fever in Northeastern Kenya, 1997-98. Emerg. Infect. Dis. 8, 138-144 (2002).

11. Balkhy, H. H. \& Memish, Z. A. Rift Valley fever: an uninvited zoonosis in the Arabian peninsula. Int. J. Antimicrob. Agents 21, 153-157 (2003).

12. Gerner, E. W. \& Meyskens, F. L. Polyamines and cancer: old molecules, new understanding. Nat. Rev. Cancer 4, 781-792 (2004).

13. Frugier, M., Florentz, C., Hosseini, M. W., Lehn, J. M. \& Giegé, R. Synthetic polyamines stimulate in vitro transcription by T7 RNA polymerase. Nucleic Acids Res. 22, 2784-2790 (1994).

14. Mandal, S., Mandal, A., Johansson, H. E., Orjalo, A. V. \& Park, M. H. Depletion of cellular polyamines, spermidine and spermine, causes a total arrest in translation and growth in mammalian cells. Proc. Natl. Acad. Sci. U. S. A. 110, 2169-2174 (2013).

15. Pegg, A. E. Mammalian Polyamine Metabolism and Function. IUBMB Life 61, 880-894 (2009).

16. Seiler, N., Bolkenius, F. N. \& Rennert, O. M. Interconversion, catabolism and elimination of the polyamines. Med. Biol. 59, 334-346 (1981).

17. van Dam, L., Korolev, N. \& Nordenskiöld, L. Polyamine-nucleic acid interactions and the effects on structure in oriented DNA fibers. Nucleic Acids Res. 30, 419-428 (2002).

18. Loprinzi, C. L. et al. Toxicity evaluation of difluoromethylornithine: doses for chemoprevention trials. Cancer Epidemiol. Prev. Biomark. 5, 371-374 (1996).

19. Milord, F., Pépin, J., Loko, L., Ethier, L. \& Mpia, B. Efficacy and toxicity of eflornithine for treatment of Trypanosoma brucei gambiense sleeping sickness. Lancet 340, 652-655 (1992).

20. Burri, C. \& Brun, R. Eflornithine for the treatment of human African trypanosomiasis. Parasitol. Res. 90 Supp 1, S49-52 (2003).

21. Gibson, W. \& Roizman, B. Compartmentalization of spermine and spermidine in the herpes simplex virion. Proc. Natl. Acad. Sci. U. S. A. 68, 2818-2821 (1971).

22. Lanzer, W. \& Holowczak, J. A. Polyamines in vaccinia virions and polypeptides released from viral cores by acid extraction. J. Virol. 16, 1254-1264 (1975).

23. Fout, G. S., Medappa, K. C., Mapoles, J. E. \& Rueckert, R. R. Radiochemical determination of polyamines in poliovirus and human rhinovirus 14. J. Biol. Chem. 259, 3639-3643 (1984).

24. Mounce, B. C. et al. Interferon-Induced Spermidine-Spermine Acetyltransferase and Polyamine Depletion Restrict Zika and Chikungunya Viruses. Cell Host Microbe 20, 167177 (2016). 
Mastrodomenico et al.

25. Mounce, B. C. et al. Inhibition of Polyamine Biosynthesis Is a Broad-Spectrum Strategy against RNA Viruses. J. Virol. 90, 9683-9692 (2016).

26. Dial, C. N., Tate, P. M., Kicmal, T. M. \& Mounce, B. C. Coxsackievirus B3 Responds to Polyamine Depletion via Enhancement of $2 A$ and $3 C$ Protease Activity. Viruses 11, 403 (2019).

27. Mastrodomenico, V. et al. Polyamine depletion inhibits bunyavirus infection via generation of noninfectious interfering virions. J. Virol. JVI.00530-19 (2019) doi:10.1128/JVI.00530-19.

28. Kicmal, T. M., Tate, P. M., Dial, C. N., Esin, J. J. \& Mounce, B. C. Polyamine depletion abrogates enterovirus cellular attachment. J. Virol. JVI.01054-19 (2019) doi:10.1128/JVI.01054-19.

29. Cohen, S. S., Morgan, S. \& Streibel, E. THE POLYAMINE CONTENT OF THE tRNA OF E. coli. Proc. Natl. Acad. Sci. 64, 669-676 (1969).

30. Ouameur, A. A., Bourassa, P. \& Tajmir-Riahi, H.-A. Probing tRNA interaction with biogenic polyamines. RNA N. Y. N 16, 1968-1979 (2010).

31. Dever, T. E., Gutierrez, E. \& Shin, B.-S. The hypusine-containing translation factor elF5A. Crit. Rev. Biochem. Mol. Biol. 49, 413-425 (2014).

32. Schnier, J., Schwelberger, H. G., Smit-McBride, Z., Kang, H. A. \& Hershey, J. W. Translation initiation factor $5 \mathrm{~A}$ and its hypusine modification are essential for cell viability in the yeast Saccharomyces cerevisiae. Mol. Cell. Biol. 11, 3105-3114 (1991).

33. Olsen, M. E. et al. Polyamines and Hypusination Are Required for Ebolavirus Gene Expression and Replication. mBio 7, (2016).

34. Olsen, M. E., Cressey, T. N., Mühlberger, E. \& Connor, J. H. Differential Mechanisms for the Involvement of Polyamines and Hypusinated elF5A in Ebola Virus Gene Expression. J. Virol. 92, (2018).

35. Schreur, P. J. W. \& Kortekaas, J. Single-Molecule FISH Reveals Non-selective Packaging of Rift Valley Fever Virus Genome Segments. PLOS Pathog. 12, e1005800 (2016).

36. Hyvönen, M. T. et al. Enantiomers of 3-methylspermidine selectively modulate deoxyhypusine synthesis and reveal important determinants for spermidine transport. ACS Chem. Biol. 10, 1417-1424 (2015).

37. Pett, D. M. \& Ginsberg, H. S. Polyamines in Type 5 Adenovirus-Infected Cells and Virions. J. Virol. 15, 1289-1292 (1975).

38. Kelly, D. C. \& Elliott, R. M. Polyamines contained by two densonucleosis viruses. J. Virol. 21, 408-410 (1977). 
Mastrodomenico et al.

640

641

642

643

644

645

646

647

648

649

650

651

652

653

654

655

656

657

658

659

660

661

662

663

664

665

666

667

668

669

670

671

672

673

39. Khomutov, A. R. et al. Methylated polyamines as research tools. Methods Mol. Biol. Clifton NJ 720, 449-461 (2011).

40. Ikegami, T., Won, S., Peters, C. J. \& Makino, S. Rescue of infectious rift valley fever virus entirely from cDNA, analysis of virus lacking the NSs gene, and expression of a foreign gene. J. Virol. 80, 2933-2940 (2006).

41. Madhubala, R. Thin-layer chromatographic method for assaying polyamines. Methods Mol. Biol. Clifton NJ 79, 131-136 (1998).

42. Ivanov, I. P. et al. Polyamine Control of Translation Elongation Regulates Start Site Selection on Antizyme Inhibitor mRNA via Ribosome Queuing. Mol. Cell 70, 254-264.e6 (2018).

\section{Figure Legends}

Figure 1. Biogenic and non-biogenic polyamines support RVFV replication. Huh7 cells were treated for four days with $1 \mathrm{mM}$ DFMO and then infected with (A) RVFV or (B) LACV at MOI 0.1. Putrescine, spermidine, and spermine were added directly to the media at the time of infection at the concentration listed. Titers were determined by plaque assay at 48 hpi. (C) Chemical structures of polyamines analyzed for their ability to support viral infection of (D) RVFV and (E) LACV. Cells were treated as in (A) and (B) but at the time of infection, cells were supplemented with $10 \mu \mathrm{M}$ polyamine as indicated. Titers were determined at 48 hpi. (D) Huh7 cells were treated as in (A) and subsequently infected with CVB3 at MOI 0.1 for $24 \mathrm{~h}$. Titers were determined by plaque assay. (G) CVB3 infections were treated with $10 \mu \mathrm{M}$ putrescine (put), spermidine (spd), spermine (spm), norspermidine (nsp), and cadaverine (cad). Titers were determined at $24 \mathrm{hpi}$. Error bars represent one standard error of the mean. ${ }^{*} p<0.05,{ }^{* *} p<0.01$, ${ }^{* * *} p<0.001$ by two-tailed Student's T-test. Comparisons in (D), $(E)$, and $(G)$ are DFMO versus treatment or as indicated.

Figure 2. Biogenic polyamines enhance infectious particle production. Huh7 cells were treated for four days with $1 \mathrm{mM}$ DFMO and subsequently infected at MOI 0.1 with (A) RVFV and (B) LACV for $48 \mathrm{~h}$. Polyamines at $10 \mu \mathrm{M}$ were added as indicated the time of infection, and titers were determined by plaque assay and genome content determined by qPCR on reversetranscribed viral RNA purified from cellular supernatant. Genome/PFU ratio was calculated by dividing the relative number of genomes by the viral titer. (C) Virus prepared as in (A) was spinoculated onto coverslips, fixed, and stained with anti-Gn or anti-Gc antibody. Mock-infected cell supernatant was similarly spinoculated as a control. (D) Virus prepared as in (A) was 
Mastrodomenico et al.

674 spinoculated and stained with anti-Gn and FITC secondary. Representative images from mock-

675 infected, untreated infected, and DFMO-treated infected samples are shown in the green and

676 red channels. (E) Particles from (D) were quantified with ImageJ and compared to viral titers to

677 obtain $(F)$ particle-to-PFU ratio. Images are representative from at least three independent

678 preparations. Error bars represent one standard error of the mean. ${ }^{*} p<0.05,{ }^{* *} p<0.01$,

$679{ }^{* * *} p<0.001$ comparing untreated to treated conditions, unless otherwise specified, using a two-

680 tailed Student's T-test.

682 Figure 3. Polyamines associate with RVFV virions. Huh7 cells were treated for four days 683 with $1 \mathrm{mM}$ DFMO and infected at MOI 0.1 with CVB3, VACV, RVFV, LACV, or HRV2. At 48 hpi, 684 cellular supernatant was collected and virus pelleted through a sucrose cushion prior to analysis 685 by (A) fluorometric assay analyzing total polyamine content or (B) thin layer chromatography. 686 Individual polyamines are indicated as put (putrescine), spd (spermidine) and spm (spermine). 687 Error bars represent standard error of the mean. ${ }^{*} \mathrm{p}<0.05,{ }^{* *} \mathrm{p}<0.01$ by two-tailed Student's $\mathrm{T}$ 688 test comparing groups as indicated. Chromatogram displayed is representative of $n=3$ 689 independent experiments.

Figure 4. Polyamines interconvert upon replenishment of DFMO-treated cells. Huh7 cells were treated for four days with $1 \mathrm{mM}$ DFMO and infected with RVFV at MOI 0.1 for $48 \mathrm{hpi}$. Polyamines were added to the cells at the time of infection.At $48 \mathrm{hpi}$, cells were collected and cellular supernatant virus purified for polyamine extraction. Polyamines were then visualized in (A) purified virions and (B) cells by thin layer chromatography. (C) Cells were treated and infected as in (A) but were supplemented with cadaverine (cad) or norspermidine (nor). Polyamine content of (C) purified virions and (D) cells was analyzed by thin layer chromatography. Chromatograms are representative of three independent experiments. (E) Virus prepared as in (C) were spinoculated onto coverslips, stained with anti-Gn antibody, and viral particles quantified. Particle counts were compared to titers to obtain the particle-to-PFU ratio. Error bars represent one standard error of the mean. No significant differences were determined by two-tailed Student's T-test.

705 Huh7 cells were treated with $1 \mathrm{mM}$ DFMO for four days prior to infection with RVFV at MOI 0.1.

706 Cells were supplemented with spermidine (Spd) and (R)-3-methylspermidine (MeSpd) at $10 \mu \mathrm{M}$.

707 Viral titers were determined at 48 hpi. (B) Cells were treated and infected as in (A) but with 
Mastrodomenico et al.

708

709

710

711

712

713

714

715

716

717

718

719

720

721

722

723

724

725

726

727

728

729

730

731

732

733

734

735

736

increasing concentrations of $(R)$-3-methylspermidine. Titers were determined at 48 hpi. (C) Cells were treated and infected as in (A) and at $48 \mathrm{hpi}$, cellular supernatant was collected, virions purified, and polyamines extracted for analysis by thin layer chromatography. (D) Cells from (C) were collected and polyamine content analysed. (E) Representative electron micrographs of virus derived from untreated, DFMO-treated, or polyamine-supplemented conditions. Error bars represent one standard error of the mean.

Figure 6. Polyamines are introduced to target cells upon infection. (A) Schematic of experimental setup. (B) $293 \mathrm{~T}$ cells were treated with $1 \mathrm{mM}$ DFMO for four days and subsequently transfected with a polyamine-sensitive dual-luciferase construct. Cells were subsequently left not treated (NT), supplemented with polyamines (a $100 \mu \mathrm{M}$ mix of putrescine, spermidine, and spermine), or infected with purified RVFV as indicated. Luciferase activity was measured $24 \mathrm{~h}$ later to calculate the relative polyamine content. RVFV-DFMO is RVFV derived from DFMO-treated, polyamine-depleted cells. Mock prep indicates treatment of transfected cells with supernatant purified as with virus purification. ${ }^{*} p<0.05,{ }^{* *} p<0.01,{ }^{* * *} p<0.001$, NS - not significant using a two-tailed Student's T-test with comparisons as indicated.

Figure 7. Polyamines maintain infectivity of bunyavirus particles. (A) RVFV derived from untreated or DFMO-treated cells was incubated at $37^{\circ} \mathrm{C}$ for the indicated time, when viral titers were determined by plaque assay. (B) RVFV stock virus was incubated with increasing doses of DFMO for $24 \mathrm{~h}$ at $37^{\circ} \mathrm{C}$. (C) Virus derived as in (A) was incubated with exogenous spermidine (Spd) or spermine $(\mathrm{Spm})$ at $37^{\circ} \mathrm{C}$ for the indicated time prior to titering by plaque assay. (D) LACV derived and treated as in (A) was incubated at $37^{\circ} \mathrm{C}$ and titered by plaque assay. (E) RVFV was incubated with increasing doses of exogenous spermidine (Spd) for $24 \mathrm{~h}$ at $37^{\circ} \mathrm{C}$ prior to plaque assay. ${ }^{*} p<0.05,{ }^{* *} p<0.01,{ }^{* * *} p<0.001$, NS not significant by two-tailed Student's T-test comparing not treated (NT) conditions to DFMO. 
bioRxiv preprint doi: https://doi.org/10.1101/2020.01.23.915900; this version posted January 24, 2020. The copyright holder for this preprint (which was not certified by peer review) is the author/funder, who has granted bioRxiv a license to display the preprint in perpetuity. It is made

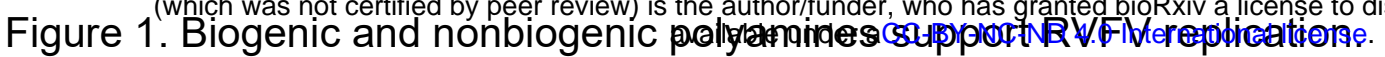

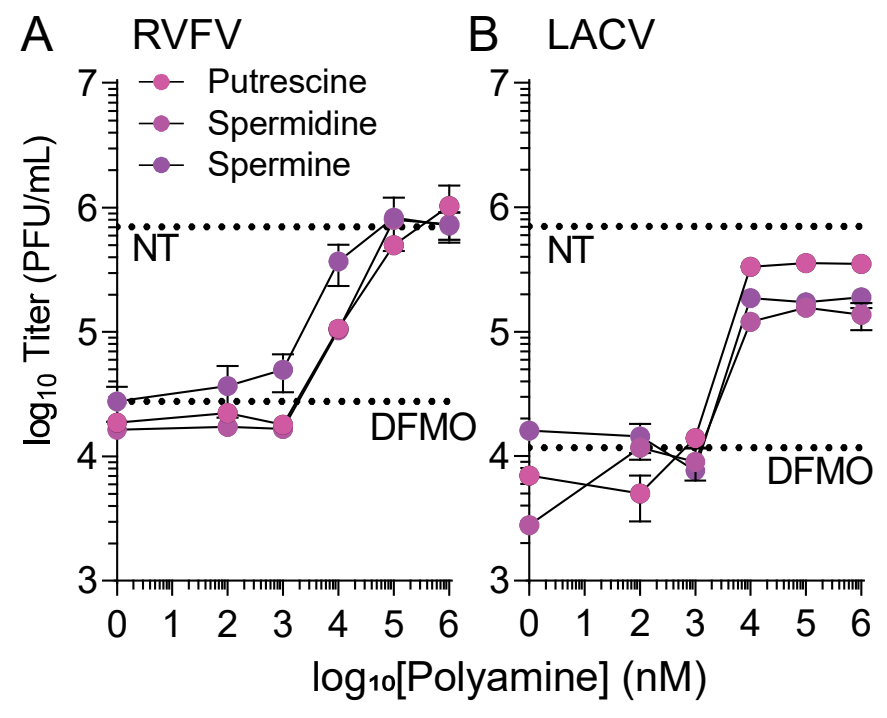

D RVFV

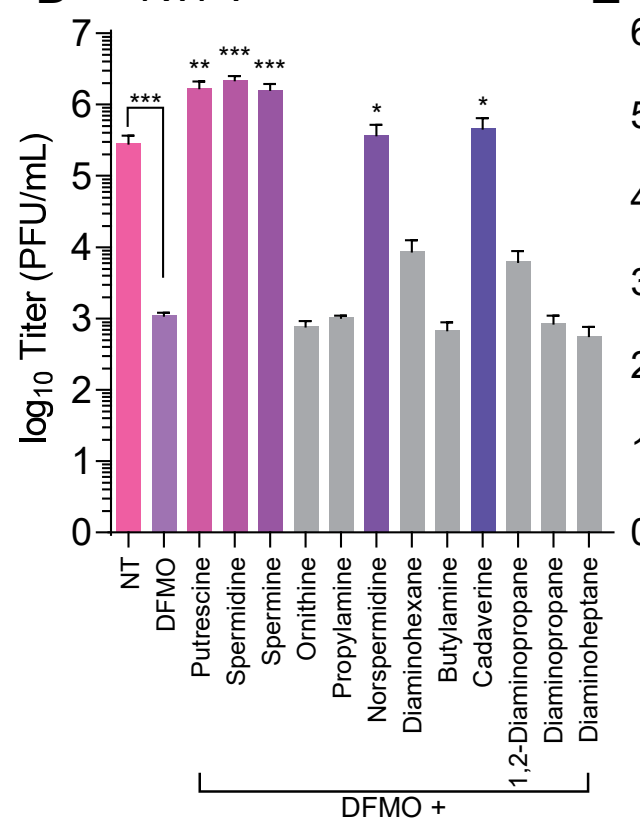

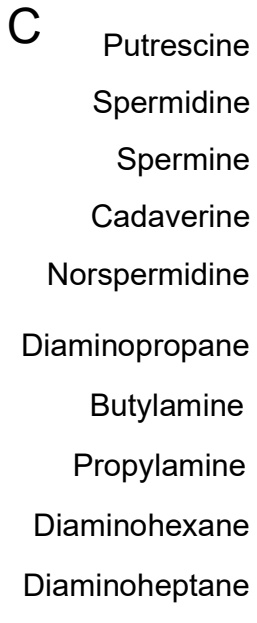

LACV

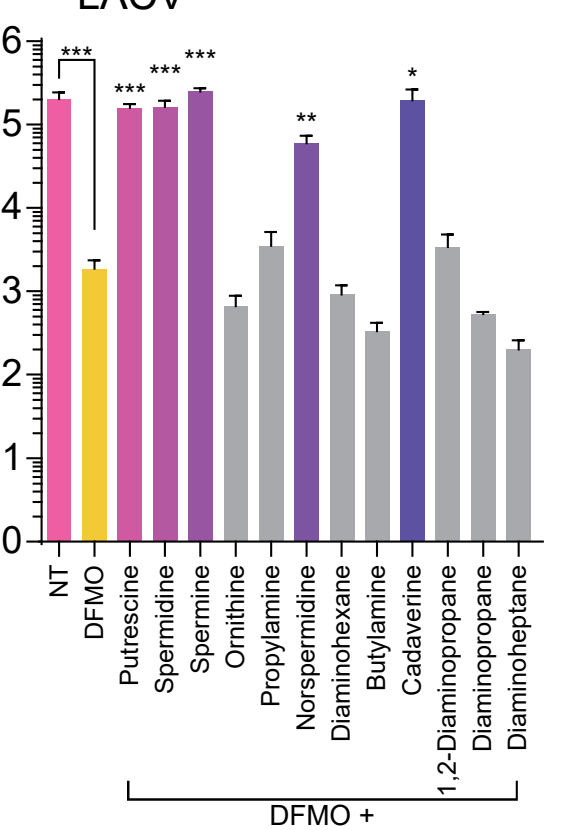

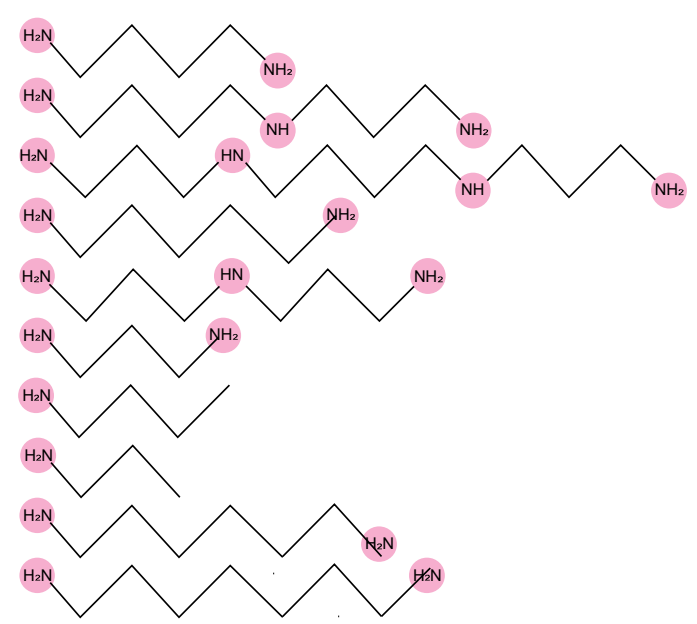
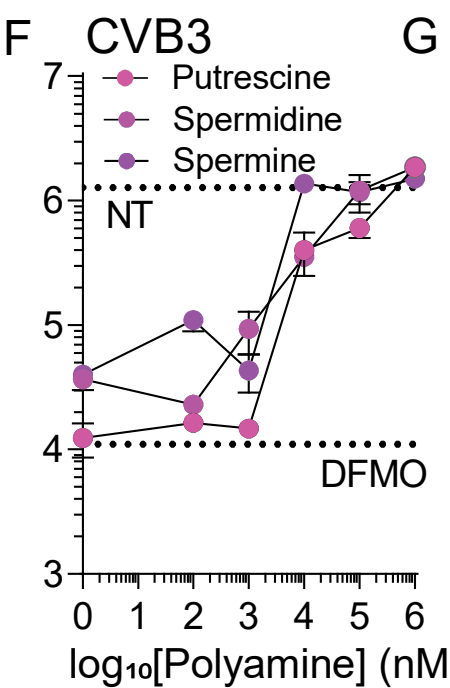

G

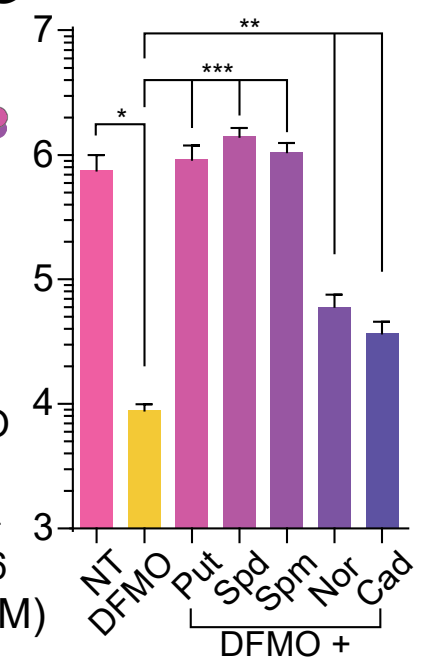



Figure 2. Biogenic polyamines evathenoed\&pecífic Niatiectivityernational license.

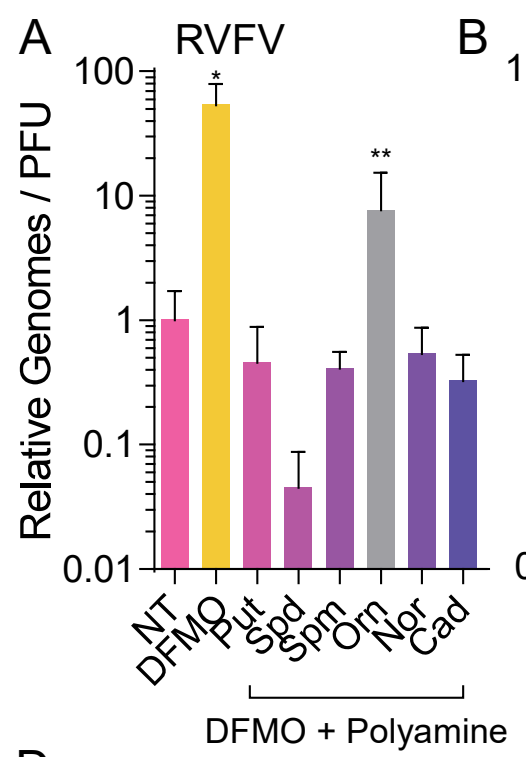

D

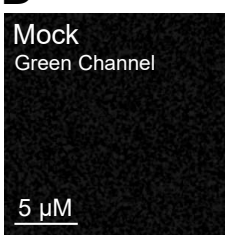

NT

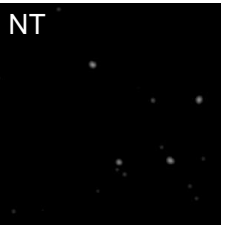

DFMO
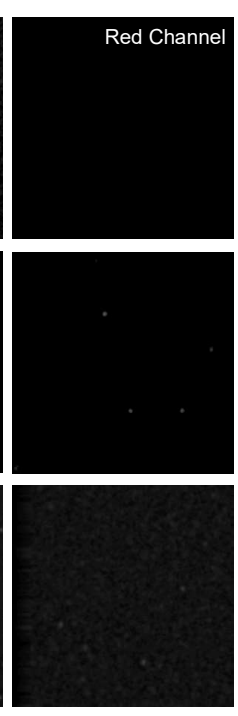

$\mathrm{B}$

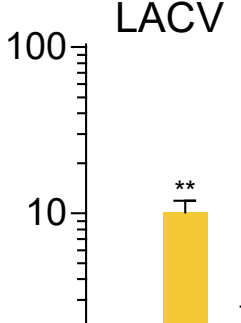

C

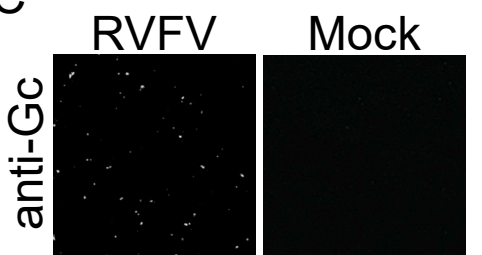

0.1
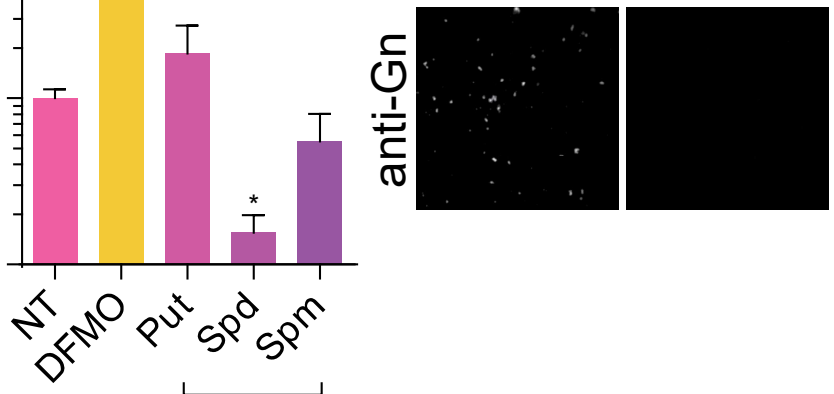

\section{$E$}

DFMO + Polyamine
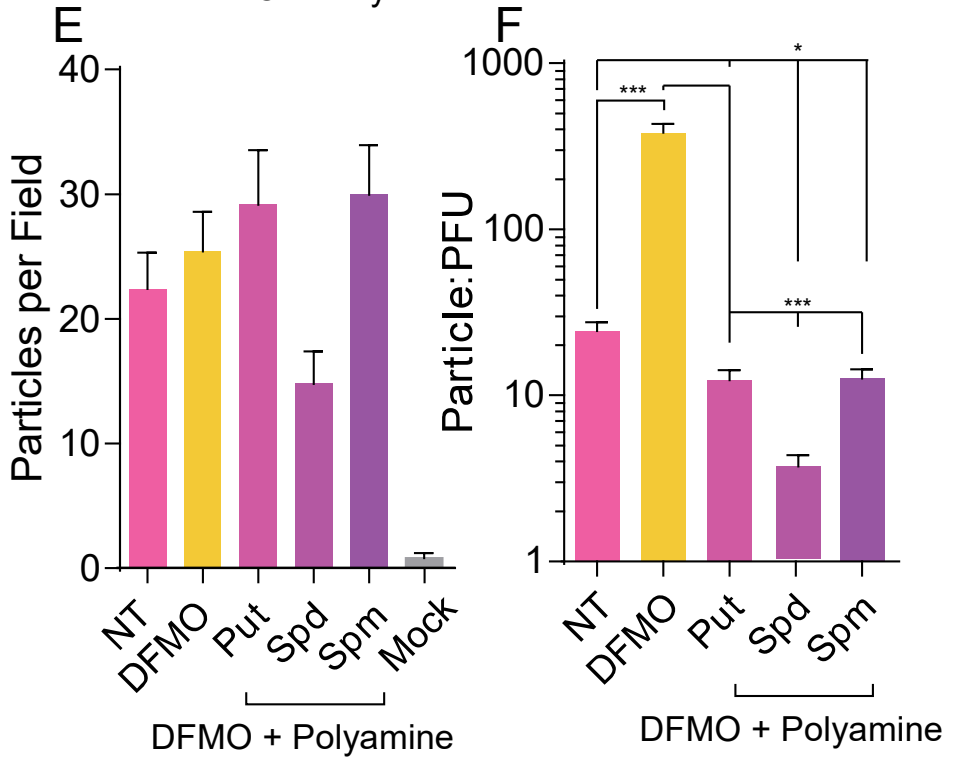
bioRxiv preprint doi: https://doi.org/10.1101/2020.01.23.915900; this version posted January 24, 2020. The copyright holder for this preprint (which was not certified by peer review) is the author/funder, who has granted bioRxiv a license to display the preprint in perpetuity. It is made

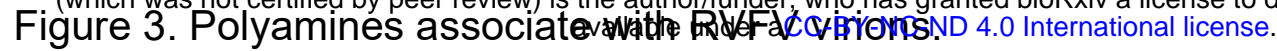
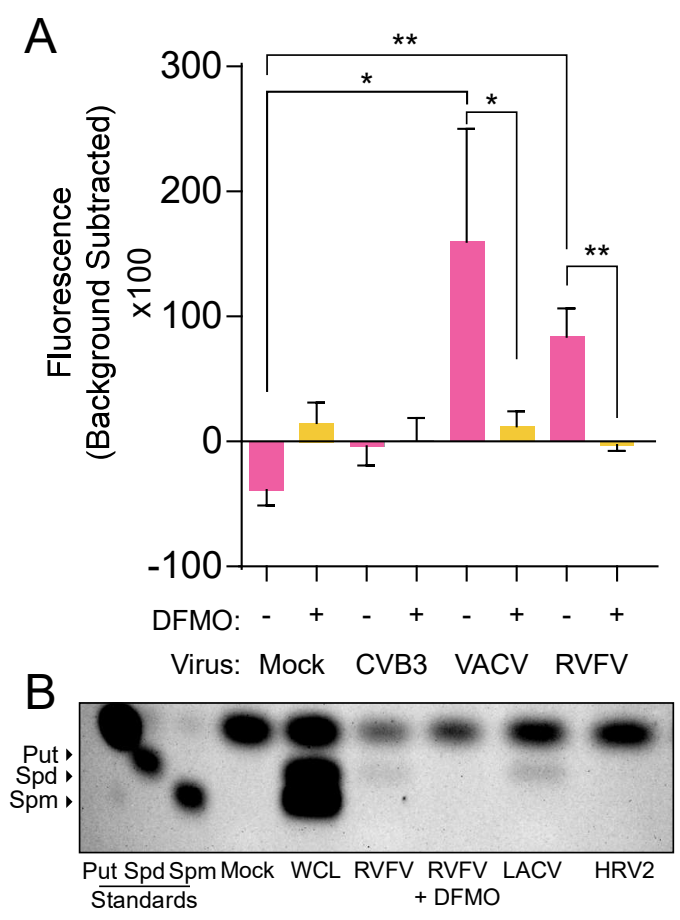
bioRxiv preprint doi: https://doi.org/10.1101/2020.01.23.915900; this version posted January 24,2020 . The copyright holder for this preprint (which was not certified by peer review) is the author/funder, who has granted bioRxiv a license to display the preprint in perpetuity. It is made

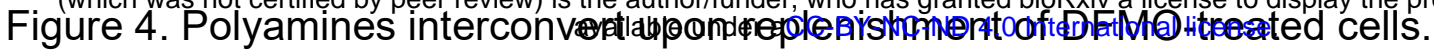
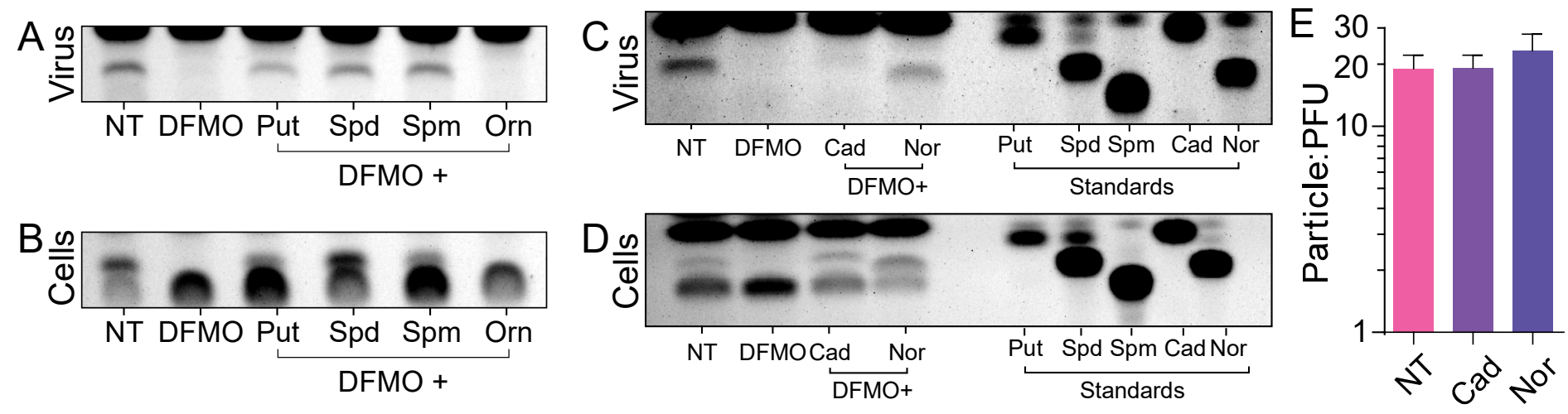


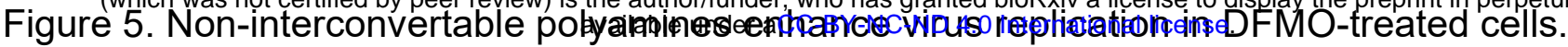

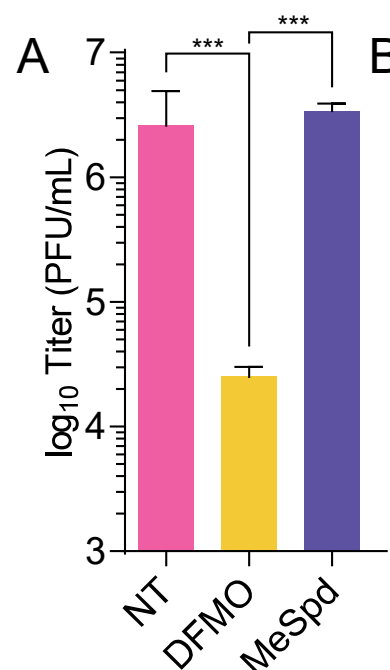

$\mathrm{E}$
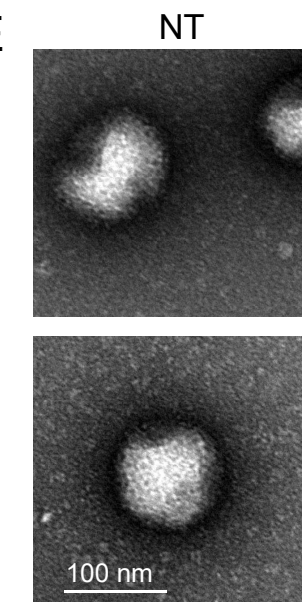

$B^{7}$ 青

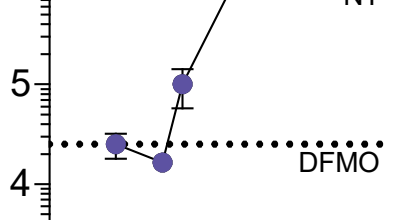

3

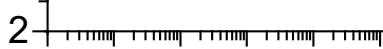

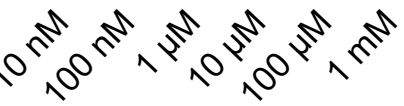

[MeSpd] added
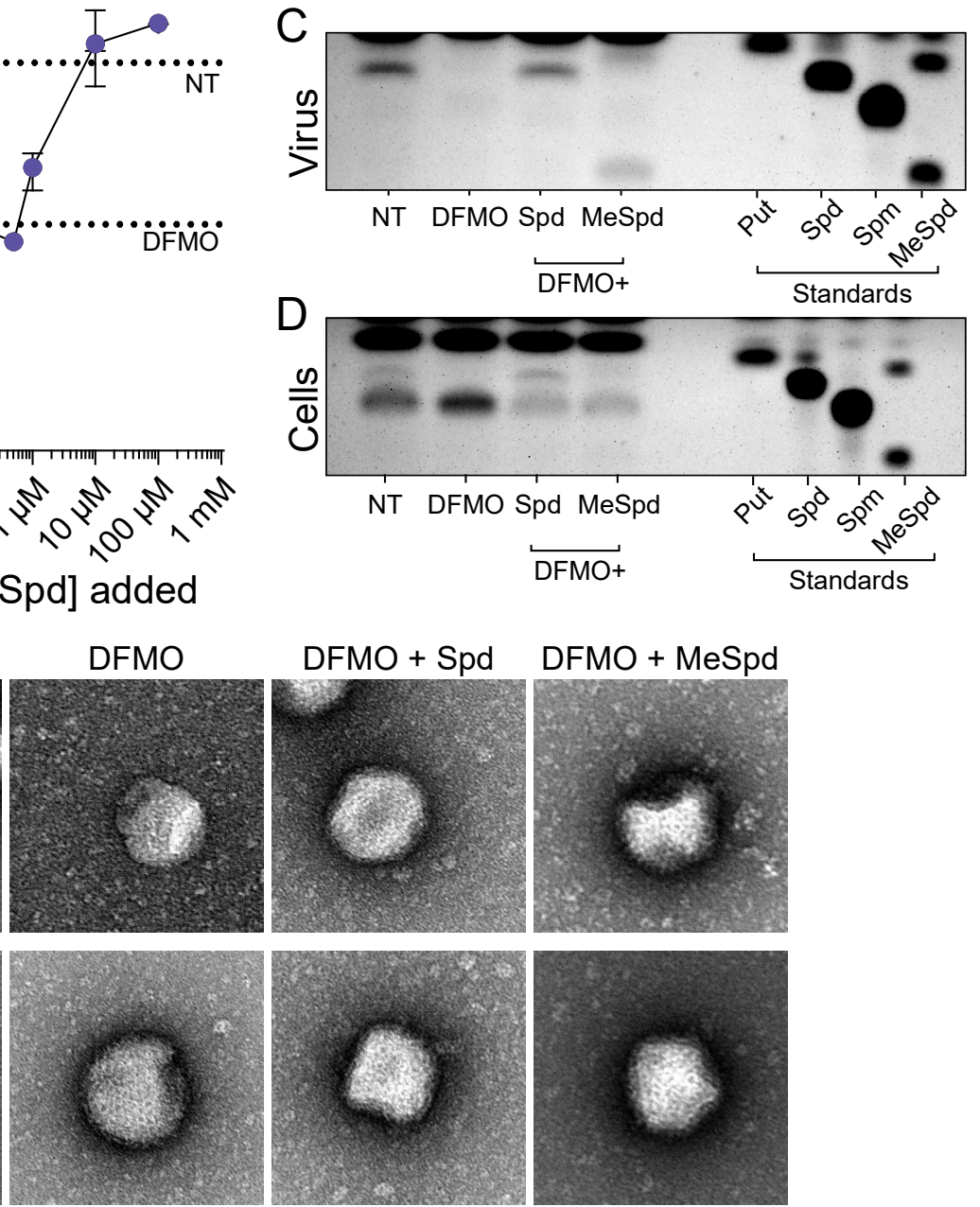
bioRxiv preprint doi: https://doi.org/10.1101/2020.01.23.915900; this version posted January 24, 2020. The copyright holder for this preprint (which was not certified by peer review) is the author/funder, who has granted bioRxiv a license to display the preprint in perpetuity. It is made

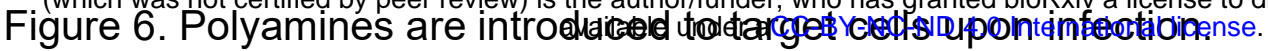

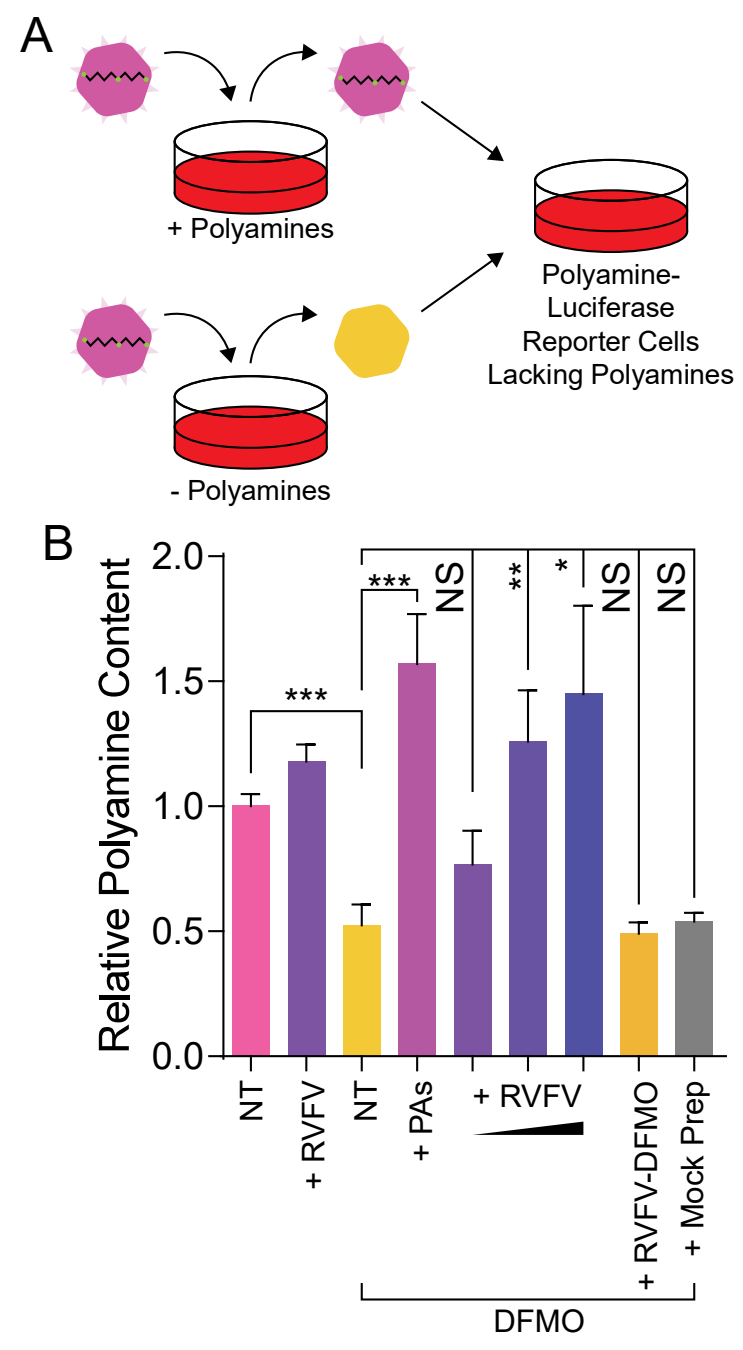


bioRxiv preprint doi: https://doi.org/10.1101/2020.01.23.915900; this version posted January 24, 2020. The copyright holder for this preprint (which was not certified by peer review) is the author/funder, who has granted bioRxiv a license to display the preprint in perpetuity. It is made

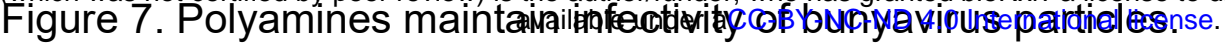
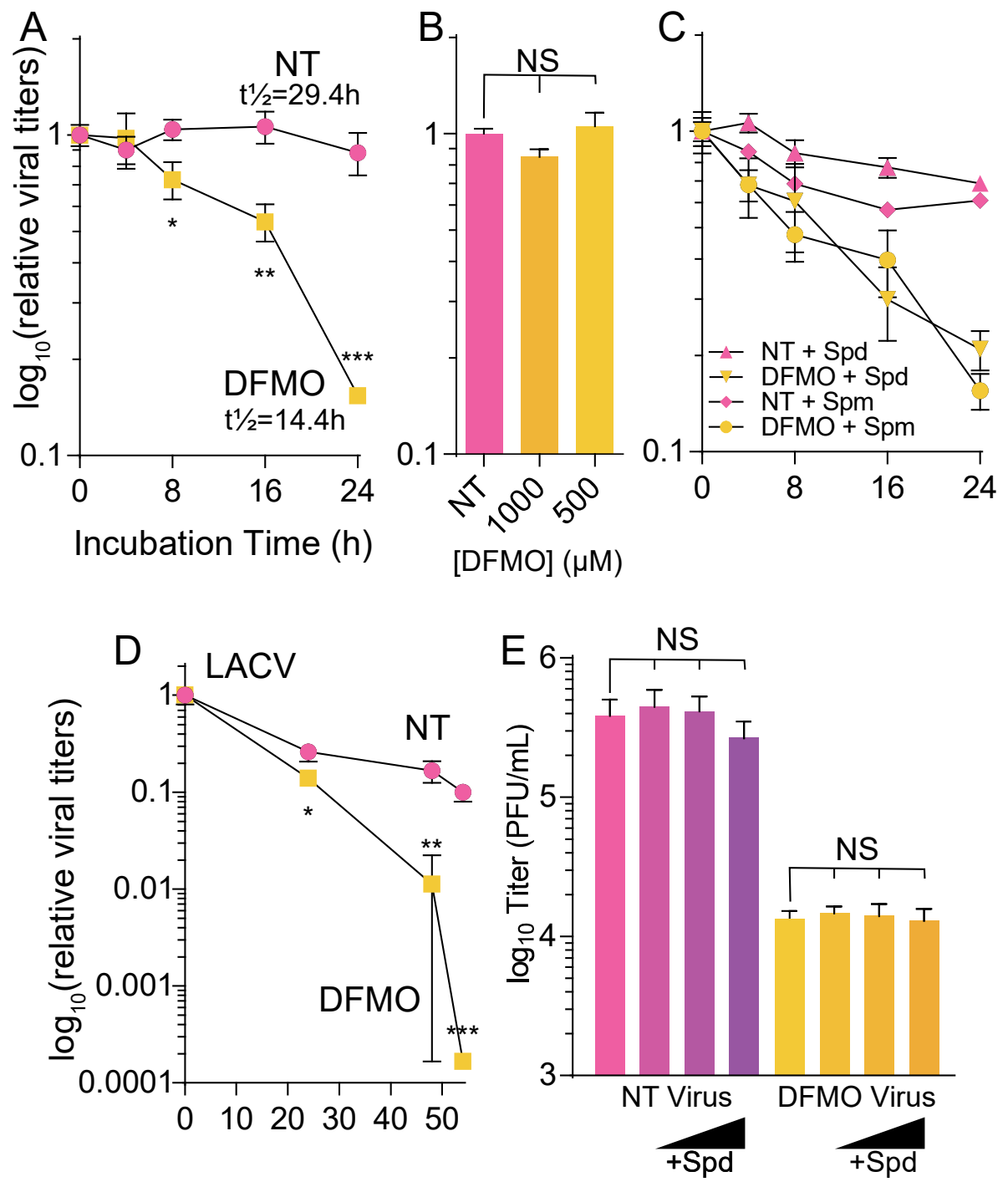